\title{
EL TEATRO: "UN ESPEJO DE LA SOCIEDAD" (Un medio para la socialización y el aprendizaje De niños (as) y jóvenes) ${ }^{(1)}$
}

\author{
Estela Socías Muñoz \\ Universidad Mayor de Chile
}

\section{RESUMEN:}

El objetivo de este trabajo es mostrar la importancia del teatro escolar en el desarrollo del niño(a) y joven durante su educación. El sentido de socialización y unión de grupos. Se utilizará el concepto de género por la importancia que cobra en lo lúdico formando seres mas acordes a la época que vivimos y con el sentido de participación conjunta sin importar, sexo, color, raza; unidos simplemente por el deseo de encontrar en el teatro un medio para exteriorizar sus sentimientos mas profundos sin temor al ridículo y logrando expresar por medio de los personajes que representan, aquellas cosas con las que sueña o con las que quiere desechar. El reconocimiento inicial de la literatura se adquiere desde los primeros años. Todos recordamos un texto que se nos contó con afán durante la infancia, así como también, nos es fácil distinguir títulos o autores de repertorio clásico que sin duda permanecen en el imaginario colectivo y que han aportado a la configuración de practicas culturales que se manifiestan en espacios públicos y privados. Es significativo añadir a estos antecedentes el concepto de género, expuesto anteriormente, por el simple hecho que en las escuelas existen numerosos casos donde se hace una diferencia notoria entre hombres y mujeres y que producen problemas en el aprendizaje sobre todo en los que recién se inician. A partir de esto, podemos considerar con mayor certeza la necesidad de mirar estos patrones desde la óptica de la cultura y como esta en todas sus manifestaciones perpetúa modelos de vida y los mantiene vigentes desde la repetición .El discurso cultural desde siempre ha expresado la diferencia entre ellas y ellos y en su conjunto la colectividad humana femenina y la colectividad humana masculina han asumido formas de apreciar el mundo, que validan sus características e invalidan las de la otredad, diferenciada por varones y mujeres; orientando la construcción de su identidad y el establecimiento de sus relaciones hacia el cumplimiento de lo que está social y culturalmente establecido. Me he referido a este concepto polisémico porque nos llevará a comprender la importancia del teatro escolar para lograr la integridad del ser humano y su comportamiento ante una sociedad que limita al crecimiento de los niños y niñas. Y veremos como el teatro puede desarrollar conductas que no significan que la condición de hombre mujer variará, al contrario los fortalecerá como seres que trabajan en forma colectiva y más humana.

\section{THEATER: "A MIRROR OF SOCIETY" (Means for socialization and learning of children and young people)}

\begin{abstract}
:
The objective of this is to show the importance of theater-school in the development of the child and young people during their education. It also discusses the meaning of socialization and union of the groups. It is used the concept of gender by its importance on requiring from the games some help for the formation of beings according to the era they are inserted in. The same concept looks on the sense of participation regardless of sex, color, and race. They (children) are simply united by the desire to find in the theater a way
\end{abstract}


to externalize their deepest feelings, without fear of ridicule and able to express through characters they represent those things they dream or want to desire.

The initial recognition of literature is acquired from the early years. We all remember a text that has been told in our childhood, as well as it is not easy to distinguish titles or authors of classical repertoire which undoubtedly remain in the collective imagination and has provided the setting for cultural practices that manifest themselves in public spaces and private ones. It is significant to add to this background the concept of gender by a simple fact that in schools there are numerous cases where there is a striking difference between men and women which produces problems in learning on all that has just begun. It is considered that the theater may develop behavior that does not mean that the condition of man or woman will vary, on the contrary, will strengthen as people who work collectively and more human.

\section{Introducción}

El reconocimiento inicial de la literatura se adquiere desde los primeros años. Todos recordamos un texto que se nos contó con afán durante la infancia, así como también, nos es fácil distinguir títulos o autores de repertorio clásico que sin duda permanecen en el imaginario colectivo y que han aportado a la configuración de practicas culturales que se manifiestan en espacios públicos y privados.

Es importante añadir a estos antecedentes el concepto de género, que las escuelas en la mayoría de los casos hacen una diferencia notoria que produce problema en el aprendizaje sobre todo en los que recién se inician.

Daremos una definición sobre el concepto de género:

Un término polisémico como es género ha adquirido desde su multiplicidad de acepciones gran importancia en los estudios culturales en las últimas décadas.

Esto, porque las reivindicaciones de espacios sociales para hombres y mujeres desde sus propias diferencias ha permanecido en alerta constante en los nuevos tiempos. Para efectos de este trabajo entenderemos el género a partir de la definición que otorga Marta Lamas:

El género es una construcción socio-cultural, que hace referencia a la identidad de mujeres y hombres, que determina sus roles y, por tanto, como deben comportarse y pensarse en sociedad nace de la diferencia de los sexos, como primera forma de oposición. (2)

Marta Lamas entiende género, de acuerdo a las diferencias que se otorgan a cada sexo, como "[...] un campo primario dentro del cual o por medio del cual se articula el poder" (3.)

Es decir, que los roles y características que se reatribuyen a cada uno, necesariamente hay intereses de poder, de un grupo dominante, por sobre otro dominado.

Según esta concepción no es el sexo propiamente tal es el que me define en cuanto a mis prácticas sociales, sino la cultura o la sociedad desde la mirada que tiene mi sexo biológico. Ocurre que esta cultura manifestará y demandará conductas correctas y erradas para hombres y mujeres que irán desde la forma de permanecerán una silla hasta como expresar sus afectos.A pesar de que ambos sexos se ven en ocasiones afectadas por dichas conductas, los cánones de vida y la mirada del mundo general que les permite reconocer la 
presión cultural o hacer la diferencia, sino que sólo asumen que ese tipo de variables son las que corresponden.

Identificar las diferencias, no es hasta ahora la marca de este trabajo, sino el punto de partida para mirar atrás luz las consecuencias de esta diferenciación de conductas desde la sexualidad. Está claro que esta dualidad hombre-mujer esta acompañado de una connotación con valoraciones positivas o negativas, ocupando la mujer el rincón de lo privado y subordinado y el hombre el espacio público y subordinante.

La posición diferenciada determinada para hombres y mujeres se construye a partir de la asignaciones de roles, espacios, características e identidades diferentes para varones y mujeres en razón del sexo biológico, que da como resultante una situación diferenciada en términos de derechos, valores y oportunidades - y un código complejo que organiza y regula las relaciones entre los sexos. (4)

A partir de esto, podemos considerar con mayor certeza la necesidad de mirar estos patrones desde la óptica de la cultura y como esta en todas sus manifestaciones perpetúa modelos de vida y los mantiene vigentes desde la repetición. El discurso cultural desde siempre ha expresado la diferencia entre ellas y ellos y en su conjunto la colectividad humana femenina y la colectividad humana masculina han asumido formas de apreciar el mundo que validan sus características e invalidan las de la otredad.

Si lo anterior, lo pensamos en términos fisiológicos, hay una oposición entre" nosotros y Ellas"; característica propia de la ideología, que según sugiere Van Dijk, "Los grupos construyen una imagen ideológica de si mismos y de los otro sexos, de tal modo que (generalmente) nosotros estamos representados positivamente y Ellos negativamente"

Esto con la finalidad de legitimar discursos y prácticas, de un grupo sobre otro, perpetuando intereses particulares.

Como la otredad ha definido supremacía en una esfera es que me detengo en el análisis de la problemática de género en la mujer. Creo en la importancia de entender la subjetividad femenina, a partir de lo que género significa para aquellas construcción .Para ello tomado ciertos elementos constituyentes que se irán desarrollando a partir de este apartado teórico.

El súper yo femenino, según Bleichmar, estaría constituido a partir del período preedíptico; y sus contenidos son el orden moral, reglamentando las relaciones con los otros, sentenciando conductas, etc., y en el narcisismo del Yo que estimula la autoestima con realizaciones de acciones reconfortantes para si según Levinton:

[...] así en ambos casos en el mandato superyoico estará presente el temor por la amenaza de una sanción: bien por la necesidad de pagar con culpa la transgresión de una norma o bajo la forma de desaprobación narcisista por la pérdida de amor por parte del súper yo. Su cumplimiento moviliza diferentes tipos de angustias. (6)

Es decir, lo que se cree acerca de lo que debiera ser una "niña-mujer" y "un niñohombre", como creencias ideológicas fuertemente enraizadas, se proyecta tempranamente en ellos por los padres, preparándose así, su correspondiente siguismo identitario. Y por consiguiente, la valoración de la norma como también, la angustia que provoca su falta.

Referirnos a sexo, es, por tanto distinto hacerlo de Género mientras que el sexo alude a lo biológico del ser humano, es decir, uno nace varón (macho) o mujer (hembra) con determinadas características, condiciones y rasgos anatomo - fisiológicos, el Género lo hace a características, roles, espacios y rasgos de personalidad que la sociedad asigna a varones y mujeres en función de su sexo y las relaciones entre ambos. Esta asignación se 
da claramente diferenciada par varones y mujeres, orientando la construcción de su identidad y el establecimiento de sus relaciones hacia el cumplimiento de lo que está social y culturalmente establecido. (7)

En todas las parcelas de la vida se manifiesta el género, toda acción estará determinada por éste, porque la repetición cultural lo hará intrínsico al niño a niña desde sus primeros años, hasta que estas conductas dejen de ser discutibles, sencillamente son. En palabras de María del Pino:

[...] desde el inicio de la vida se le enseña y exige modelos de comportamientos ligados a su sexo, lo que se manifiesta en infinidad de comportamientos ligados a su sexo, Lo, que se manifiesta en infinidad de circunstancias del entorno familiar (en el lenguaje en el color de la ropa, en las recriminaciones -"Los niños no lloran ", las niñas no juegan con pistolas",[...]se oye decir a padres, incluso en orientaciones para la elección de los amigos/as no están exentas de connotaciones de génerono vayas con ese niño que es amanerado" o esa niña no me gusta porque se comporta como un niño". Esta es la manifestación predominante que la escuela debe cambiar. (8)

Me he referido a este concepto polisémico porque nos llevará a comprender la importancia del teatro escolar para lograr la integridad del ser humano y su comportamiento ante una sociedad que limita al crecimiento de los niños y niñas.

$\mathrm{Y}$ veremos como el teatro puede desarrollar conductas que no significan que la condición de hombre mujer variará, al contrario los fortalecerá como seres que trabajan en forma colectiva y más humana.

Ahora llevando estos conceptos al área dramática motivo de este trabajo, el escritor cubano Luís Cabrera en su libro "teatro escolar" expresa textualmente "si nos referimos al teatro se remonta a los lejanos tiempos de la formación de la sociedad humana y su germen hay que buscarlo en el animismo y en la magia. (9)

En este contexto, el teatro como medio de comunicación es un lazo social, y por tanto nos mostrará la realidad que vivimos en un momento determinado.

Estoy convencida que en el teatro el niño (a) o joven encontrará el estímulo para poder proyectarse en los conocimientos que va aprendiendo, además los objetivos fundamentales transversales quedan demostrados, ya que conjugan todo tipo de materias que se enseñan en los establecimientos educacionales, tales como poesía, danza, música, pintura, vestuario, fotografía, textos literarios y no literarios, es decir la proyección en el ser humano está absolutamente comprobada.

Pensando en la necesidad de hacer del teatro un medio de socialización de los alumnos y alumnas este estudio intentará encontrar en la dramaturgia, y en el teatro de títeres, una forma lúdica de adquirir conocimientos en un arte como lo es el teatro escolar que aún tiene muchas dificultades para formar parte importante del currículo que los maestros deben aplicar como parte de los planes de educación que imparte el gobierno en este estudio se pretende convocar este tipo de conocimiento como una poderosa fuente de transmisión cultural en los espectros más cotidianos y en los más complejos.

Haciendo presente además la importancia que tiene en la formación de un grupo socialmente unidos sin discriminaciones de sexo, raza y cultura y valorizando las condiciones de cada uno, respetando siempre las limitaciones que los seres humanos poseemos desde el nacimiento. 


\section{Teatro en la escuela}

Existen desde los tiempos más remotos diferentes disciplinas que convierten al teatro como una actividad que utilizará diferentes metodologías de acuerdo al objetivo que se persigue. textualmente:

Según Luís Cabrera, escritor cubano en lo que se refiere a teatro en la escuela dice

La concepción tradicional del teatro es la que se debe aplicar en la enseñanza de los niños(as) jóvenes, que ellos hagan representaciones, sin tener en cuenta que el teatro, como expresión artística que es, responde al disfrute y enriquecimiento espiritual del individuo, y para ello, en el niño nada mejor que el juego, independientemente que el teatro en sí mismo no es más que un divertimiento. (10)

Los niños desarrollan desde la más temprana edad, el interés por imitar lo que ellos van incorporando a su imaginario colectivo, ya sea en el comportamiento con sus pares, o cuando se relacionan en los juegos, o simplemente cuando se encuentran frente a un aparato de televisión horas entreteniéndose, constituyéndose por ende un profesor que en muchos casos es peligroso

Estela Socías, Pamela Césped cuando expresan en el seminario para titulación de profesora básica "La televisión abierta es un profesor peligroso, puesto que transmite costumbres, lenguaje inapropiado de los niños y niñas que permanecen frente a una pantalla como un medio de entretención. (11)

Con los antecedentes antes expuestos debemos considerar al teatro escolar como un proceso formativo, además el hecho que en esta actividad involucran a profesores, alumnos y padres, estos últimos sentirán que sus hijos al estar representando una obra en un escenario son actores y los acompañan desde el aprendizaje, hasta en el vestuario, eso hace que los educandos se incentiven a leer y comprender los textos que tienen como programa durante el año escolar de una forma lúdica y entretenida, además los hará cuestionarse como receptores críticos los programas que ven en la TV, puesto que el hecho de participar activamente en representaciones, los convierte en personas menos fácil de convencer con vulgaridades, y lenguaje mal empleado.

En esto debemos tener presente que esta actividad no está plenamente desarrollada a nivel escolar, ya que la mayoría de los casos los profesores la utilizan para que los niños y niñas reciten poesías y textos literarios de acuerdo a lo que ellos consideran parte del aprendizaje, dejando a un lado la parte más importante que se debe considerar dentro del teatro que es la improvisación, la soltura, el cambio de expresiones, la libertad en el desplazamiento, en el fondo se impone el mundo de los grandes.

Además debemos considerar que en la primera etapa los niños, no saben leer, por lo tanto los textos no podrán ser actuados de una forma rigurosa, es por eso que es necesario utilizar una metodología diferente, es decir combinar juego con la estética en los movimientos en el color, en la forma y en la música. En cuanto a los más jóvenes se debe utilizar aquellas caracterizaciones que vayan de acuerdo a su edad. Y que casi siempre no tiene mayor diferencia con el teatro de los adultos. 
Es importante que los maestros que fomenten el teatro en sus clases de lenguaje y comunicación, deban conocer de él, sus técnicas la sensibilidad que este tipo de arte tiene como objetivo principal.

A mi juicio los profesores durante el curso de sus carreras educativas, reciben instrucciones acerca de lo que es en si el teatro, por lo tanto, pueden manejar en forma positiva el lenguaje y la expresión, no obstante en las Universidades que imparten la carrera de docencia deberían tener presente que el teatro escolar, debe ser más que un ramo optativo, ya que lo que se pretende demostrar en este estudio es que el teatro es un medio de socializar al niño o niña e insertándolo con sus pares durante toda la época escolar, el teatro es una practica además colectiva por lo que hará que el profesor deba implantar ciertas normas, con el fin de los alumnos ( as)adquieran un orden y disciplina y respeto entre ellos, que les servirá para lograr en todos los demás ramos una unión de todos los que participan de un curso y podrán aplicarlo en todas los demás ramos, es por eso que en este punto hago hincapié en la importancia de la dramaturgia en la enseñanza.

El profesor tendrá además la responsabilidad de entregar a cada alumno el papel que le corresponderá representar, habiendo hecho previamente y con el conocimiento de cada uno de sus alumnos una selección de roles que la obra requiera. (En el fondo está indicando que deberá formarse una idea global de la personalidad de cada uno de sus alumnos)

En la primera etapa el profesor deberá escoger textos fáciles, con papeles y roles bien determinado para que los educandos vayan acostumbrándose a su participación dentro del grupo escogido, y pierdan muchos de ellos el pánico escénico, que se da mucho en los alumnos de la básica por temor a hacer el ridículo.

Como podemos ver en el desarrollo de este tema, hay muchos objetivos que los profesores deben realizar, conocimiento de cada uno de sus alumnos, enseñarles disciplina sin ser autoritarios, que el niño se sienta libre en un mundo donde la creación va de la mano con la imaginación, sin fomentar la diferenciación marcada de sexo, porque en lo lúdico y fantástico todo está permitido, colores, comportamientos y las representaciones solo son una forma de expresión.

Según el escritor Luís Cabrera en su texto" Teatro Escolar" describe qué en el hay ciertos requisitos que deben tener presente los educadores:

Los requisitos son tres:

requisitos teatrales, psicológicos y pedagógicos" refiriéndose al primero dice" teatrales, "el material escrito que el niño representará debe tener todas las posibilidades que hacen teatral una obra cualquiera "Psicológicos, descartará el miedo como resorte para despertar o avivar el interés, utilizará la magia como elemento poético, pero no para resolver situaciones, No se valdrá de la de la mentira ni del engaño y evitará las identificaciones que puedan ir en desmedro de la personalidad del niño o niña, no ridicularizará figuras como profesores, padres abuelos, etc. por último el final en el caso de los mas pequeños deberá ser feliz. Por último en el aspecto pedagógico el vocabulario debe estar de acuerdo con el nivel de cada uno de los alumnos o (as). (12)

Ahora abocándonos en el tema que se pretende demostrar es necesario hacer algunas observaciones que tienen que ver con la experiencia adquirida en prácticas que he realizado en colegios a lo largo de varios períodos escolares.

El niño que recién empieza su escolaridad, necesita movimiento, espacios libres, si bien no capta lo que es la libertad pero si se da cuenta que en una jaula un pajarito está 
llorando para que le abran la puerta, y cuando logra y vuela, el niño se encanta al ver como se desliza por el cielo, es importante darnos cuenta que ya en ese instante tiene un concepto claro acerca de lo que le produce motivaciones. Es necesario ir desarrollando en ellos, los colores la musicalidad, los animales que hablan que tienen aventuras.

Por otro lado los niños (as) necesitan comunicarse con sus pares, es ahí donde empieza su socialización y a veces su timidez, algunos complejos que traen desde sus casas, los hace menos receptivos a expresar sus emociones, por eso al crear personajes, tales como muñecas con vida, animales, que bailan, por eso es necesario que realicen todo este tipo de acciones que en ellos son naturales, pero que se transforman en reales cuando un adulto los interrumpe con preguntas, y se inhiben, por eso es importante hacer del juego un instrumento que los conducirá a realizar actividades en su mundo imaginario, desde ese punto los educadores debemos encauzarlo a lo que llamamos teatro.

Luís Cabrera en su libro Teatro Escolar expresa:

Si por un lado el juego infantil tiene elementos de la representación, el teatro por su lado, tiene elementos de juego, ya que una representación no es otra cosa que un juego de la realidad, de ahí que los juegos infantiles y el teatro sea el sostén o punto de partida de los juegos escénicos. (13)

Esto nos está dando a entender que todo lo que vivimos diariamente, son historias, y que por ende son plausibles de llevar a la practica teatral, mediante representaciones que nos mostrarán la realidad del momento, donde pondremos magia y que serán representadas por los propios alumnos a los que se les asignará un rol.

Además será una actividad donde compartirán todos los estudiantes de un curso determinado.

Es de gran importancia que estas actividades se realicen en el lugar donde se inició la acción, de esta forma no producirá distracción y el ambiente les será familiar.

\section{III.- La Magia de los títeres.}

El niño está abierto como se ha dicho al juego, los títeres han representado siempre un elemento mágico que hace real el mundo de la fantasía, reproducido en este caso por títeres, marionetas , muñecos de trapos.

El escritor y Profesor peruano Danilo Sánchez Lión en el I festival de títeres en la educación el día 13 de Diciembre 2008, expresaba:

El espectador animista natural del teatro de títeres es donde acepta que hasta una piedra hable, cante y baile. El teatro de títeres se vincula el alma infantil por la capacidad de poder abordar de manera alegórica aspectos esenciales de la vida y poder hacerlo de manera directa y verdadera sin las hipocresías ni prejuicios de que es esta imbuida el alma de los adultos. (14)

Mediante los títeres podemos llegar a captar los más íntimos sentimientos de un niño, podremos observar aquellas cosas que lo afectan, pues no son ellos lo que hablaran directamente si no que sus títeres, ellos contarán las alegrías, penas, aventuras, derrotas etc. Es por eso que es necesario observar cada gesto, palabra, acción que ellos le van dando a 
su muñeco, de esa manera podremos motivarlos a que puedan compartir con sus compañeros sus emociones.

El tiempo que se tiene en este tipo de encuentro es corto, y por lo tanto deberemos ir llegando a conclusiones que realmente nos ayuden a comprobar en la practica que el teatro infantil, el teatro de títeres, los muñecos de trapo son un medio de socializar a los niños y un medio de poder obtener en forma lúdica todos los conocimientos.

De lo expuesto podemos concluir. Que tanto el teatro escolar como el teatro de títeres, marionetas juegos con muñecos de trapo, constituirá un método lúdico de aprendizaje, donde queda establecido que el niño logrará mediante este tipo de acciones, o actividades, socializarse, compartir con sus compañeros, expresar libremente sus emociones, y harán de la clase un motivo de entretención, donde no quedará espacio para el aburrimiento ni la violencia

Como el teatro es una actividad colectiva, podrá lograrse un espacio donde todos compartirán juegos, por lo tanto estrecharan vínculos más afectivos entre ellos, que los harán por tanto seres mas receptivos en todas las materias que la escolarización imparte.

Podrán desarrollar una serie de actividades de un fondo valórico, religioso, costumbrista que los apoyará en todas las acciones que realicen tanto en el ámbito estudiantil como en sus hogares y barrios y conformarán un grupo humano unido y respetuoso de sus pares y sus limitaciones.

Concluyo con las palabras de Gabriel García Márquez:

Si por un instante Dios se olvidara

De que soy una marioneta de trapo

Y me regalara un trozo de vida

Posiblemente no diría todo lo que pienso,

Pero, en definitiva, pensaría todo lo que digo.

\section{V.-Citas}

* - Estela Socías Muñoz, Presidenta de la academia Chilena de literatura infantil-juvenil (Achli), Docente, Magíster en Literatura, Escritora

1.- $\quad$ Ponencia presentada en Talleres Jornadas Educacionales Feria del Libro 2009

2.- Lamas, M "Usos, Dificultades y posibilidades de la categoría de Género", en Compilación: El Género: La Construcción Cultural de la diferencia sexual Op. cit., p.3.3.3.1

3.- Ídem

4.- Cfr.De Barbieri,T (1992) sobre la categoría de género. Una introducción, teórica metodológica. Fin de siglo género y cambio civilizatorio, Edición de la mujer $\mathrm{N}^{\mathrm{o}}$ 17, pp.13-14

5.- $\quad$ Van Dijk, T Ideología: Un enfoque Multidisciplinario, p .15

6.- $\quad$ Van Dijk, T ídem, p.16

7.- $\quad$ De Barbieri, T. Op. cit ., p,14

8.- $\quad$ Del Pino L., María Op, cit., p, 37

9.- Cabrera, Luís escritor “Teatro en la escuela”.Olmué Ediciones. sep.2005

10- Cabrera, Luís escritor texto" Teatro en la Escuela”.Pág.101

11.- Estela Socías y Pamela Césped Seminario de título para optar al grado de profesora educación básica mención lenguaje y comunicación. Universidad Mayor Pág.53 
12.- Luis Cabrera. Texto Teatro Escolar, Pág. 25

13.- Ídem

14.- Daniel Sánchez Lión, escritor y profesor

\section{IV.-Bibliografía}

CABRERA, Luís texto “Teatro en la Escuela” Olmué Ediciones. Septiembre 2005.

SOCIAS, Estela; CÉSPED, Pamela. Seminario para optar al grado de Profesora de Educación Básica Mención Lenguaje y Comunicación. Diciembre 2008.

SÁNCHEZ, Danilo, Profesor, escritorwww.danilodasnchezlihon.bolgspot.com

Artigo recebido em: 01/12/2009

Aprovado em: 04/04/2010 